\title{
Humboldt y el rayo del Catatumbo: ¿mito o realidad?
}

\author{
가 Horacio Torres-Sánchez \\ Profesor Emérito de la Universidad Nacional de Colombia
}

\begin{abstract}
Resumen
Este articulo presenta a la comunidad académica internacional los resultados de varios años de investigación sobre el fenómeno del rayo, dentro del grupo de investigación PAAS-UN de la Universidad Nacional de Colombia, específicamente sobre el llamado rayo del Catatumbo. Este fenómeno meteorológico se presenta desde la zona sur del lago de Maracaibo en Venezuela hasta la cuenca inferior del rio Catatumbo y más allá, en Colombia, de donde procede su nombre. El fenómeno tiene un interés histórico por saber cómo el explorador prusiano Alexander von Humboldt supo de su actividad en sus exploraciones a finales del siglo XVIII y un interés científico porque hoy en día se sabe que la región del Catatumbo presenta la mayor actividad de rayos del mundo.
\end{abstract}

Palabras clave: Rayo; Alexander von Humboldt; Catatumbo.

Humboldt and the Catatumbo's Lightning

\begin{abstract}
This article presents to the international academic community the results of several years of research on the phenomenon of lightning, within the PAAS-UN research group of the National University of Colombia, specifically the so-called Catatumbo's lightning. This meteorological phenomenon occurs from the southern area of Lake Maracaibo in Venezuela to the lower basin of the Catatumbo River and beyond, in Colombia, where its name comes from. The meteorological phenomenon has a historical interest associated to Prussian traveler Alexander von Humboldt who learned of his activity during his explorations at the end of the $18^{\text {th }}$ century, and a scientific interest, as it the Catatumbo region is considered to have the highest lightning activity in the world.
\end{abstract}

Key Words: Lightning; Alexander von Humboldt; Catatumbo

\section{Introducción}

El rayo del Catatumbo es un fenómeno meteorológico que se presenta desde la zona sur del lago de Maracaibo en Venezuela hasta la cuenca inferior del río Catatumbo y más allá, en Colombia, de donde procede su nombre. De acuerdo con resultados de investigaciones recientes es la zona de mayor actividad de rayos del mundo.

Se afirma recurrentemente que el naturalista y explorador prusiano Alexander von Humboldt (1769-1859) lo describió como "explosiones eléctricas que son como fulgores fosforescentes..." ¿Qué tanto de mito o de realidad tiene esta afirmación de Humboldt?

Para responder esta pregunta me he basado, fundamentalmente, en los escritos de Humboldt incluidos en el Viage a las regiones equinocciales del nuevo continente, 17991804, Vols. I, II, III, IV, V, escrito originalmente en francés por Humboldt en París, entre 1804 y 1827. Asimismo, existe una traducción de los originales por el venezolano Lisandro Alvarado de 1941 a 1956, a partir de "Humboldt et Bonpland. Voyages dans les régions équatoriales du nouveau continent fait dans les années 1799 a 1804".
El titulo de sus escritos: "regiones equinocciales" es algo confuso, pues el equinoccio es el momento del año en que el Sol forma un eje perpendicular con el ecuador y en el que la duración del día es igual a la de la noche en toda la Tierra. Los equinoccios son el instante (o la fecha, en un sentido más general) en que suceden determinados cambios estacionales, opuestos para el hemisferio norte y el hemisferio sur. Por tanto, creo que, aunque el título de sus escritos es algo confuso, Humboldt se refería a la región tropical del continente americano.

El viaje. El mapa de la figura 1 representa el itinerario de Humboldt entre 1799 y 1804, lo cual nos servirá de referencia para conocer en donde estuvo presente Humboldt con respecto a la región del Catatumbo.

Aportes de Humboldt a la ciencia física. Humboldt no aportó nada a la física del electromagnetismo. Tal como si lo hicieron, por ejemplo, Franklyn, Volta, Oersted,

\footnotetext{
Correspondencia:

Horacio Torres-Sánchez; htorres@gmail.com

Recibido: 6 de septiembre de 2019

Aceptado: 11 de septiembre de 2019

Editor: Alberto Gómez
} 


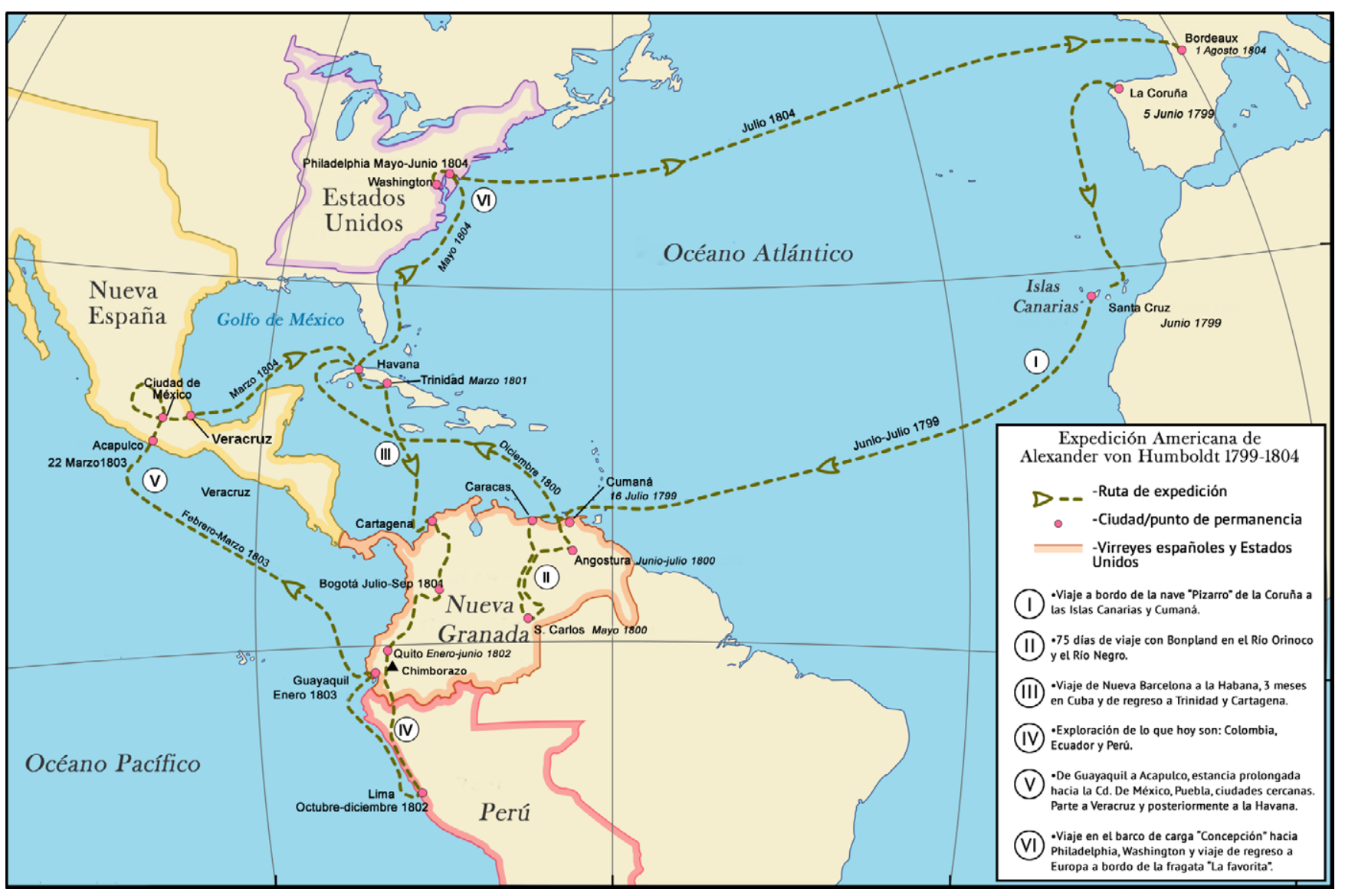

Figura 1. Itinerario de Humboldt en su viaje a la region tropical de America. Tomado de: https://www.eje21.com.co/2019/08/humboldt-ysu-periplo-cientifico-por-america/

Faraday, Coulomb, Maxwell o Jean-Baptiste Boussingault, informador de Humboldt en sus expediciones en el ejército de Bolívar, descubridor de la fijación del nitrógeno en los suelos, fundamental para el entendimiento de la función de un rayo para formar óxidos de nitrógeno a partir del nitrógeno atmosférico y posteriormente nitratos.

Sin embargo, Humboldt aportó observaciones, descripciones, mediciones, diferencias comparativas en fenómenos naturales, pero, como muy bien lo explicita en uno de sus escritos: "Lejos estoy de buscar la causa física de estas diferencias...". El gran aporte de Humboldt es su pensamiento, su manera de trabajar que relacionaba todo con todo, lo que hoy en día conocemos como interdisciplinariedad, ya que fue geógrafo, astrónomo, humanista, naturalista, explorador y muy interesado en los avances de la época sobre electricidad.

Humboldt tuvo mucho interés en las nuevas explicaciones sobre el fenómeno de la electricidad, cuando escribió: "He reunido en esta obra las experiencias de Gimnotos sobre la acción Eléctrica" (Tomo I, pg. xxxiv). Humboldt se refiere a un ensayo que publicó en 1796: Sobre la irritación de la fibra muscular y nerviosa, y sobre la acción química que sostiene la vida de los animales y plantas, basado en las investigaciones de Galvani y Volta. Los gimnotos son peces eléctricos (similares a las anguilas), abundantes en América meridional.

Para la época de Humboldt, el contexto de las investigaciones sobre el fenómeno electromagnético era:

- En 1752, Benjamín Franklin (1706-1790) demostró la naturaleza eléctrica de los rayos.

- En 1776, Charles Agustín de Coulomb (1736-1806) inventó la balanza de torsión con la cual midió con exactitud la fuerza entre las "cargas eléctricas" y corroboró que dicha fuerza era proporcional al producto de las "cargas" individuales e inversamente proporcional al cuadrado de la distancia que las separa. Hoy en día Coulomb es la unidad de medida de carga eléctrica.

- Desde 1775, Alejandro Volta (1745-1827) se dedicó a la investigación sobre el fenómeno eléctrico. Se inspiró en los estudios del físico Italiano Luigi Galvani (1737-1798) sobre las corrientes nerviosas-eléctricas en las ancas de ranas. Y de allí tomó Humboldt el modelo para su ensayo sobre Gimnotos.

- Los grandes desarrollos sobre el fenómeno electromagnético como el descubrimiento del electromagnetismo con Oersted en 1819, las leyes del Electromagnetismo 
de Maxwell (1865), el descubrimiento del electrón de Thompson en 1897, la Transmisión de energía eléctrica de Tesla en 1890 y los principios generales sobre la física del rayo por Wilson en 1920, se dieron mucho después del viaje de Humboldt al Nuevo Mundo.

- Sin embargo, en su libro Cosmos (1874), Humboldt ya reconocía los avances del electromagnetismo de Oersted, Faraday y Gauss.

Mediciones eléctricas de Humboldt en América tropical. De acuerdo con la época de su viaje (1799-1804), el instrumento que utilizó Humboldt para medir la electricidad atmosférica fue el electrómetro. Humboldt le llama en sus escritos Electrómetro de Volta: "Tanteé al punto la electricidad atmosférica con el electrómetro de Volta". (Vol. II, pg. 221)

Uno de los modelos de electrómetro o electroscopio está constituido por dos hojuelas de oro suspendidas de una varilla metálica BB ' (figura 2), terminada en su parte superior por una esfera metálica B, y fija por medio de un tapón aislador en el cuello de una campana de vidrio C. Las dos varillas $c, d$ que se ven a uno y otro lado de las hojuelas $a, b$ sirven para aumentar la sensibilidad del aparato, y la capsulita que se nota en el fondo de la campana está destinada a contener una cierta cantidad de cloruro de calcio con objeto de que se mantenga seco el aire interior.

Sus planes de correlación y sus excelentes observaciones sin buscar la causa física de las diferencias encontradas. En sus escritos Humboldt planeaba conocer los fenómenos físicos, incluido el eléctrico: "He tratado reunir en un solo plan el conjunto de los fenómenos físicos que presenta la parte del nuevo continente, comprendida en la zona tórrida, desde el nivel del mar del Sur hasta la cumbre de la más elevada montaña de los Andes; a saber: la vegetación, los animales, las relaciones geológicas, la cultura del sol, la temperatura del aire, los límites de las nieves perpetuas, la constitución química de la atmósfera, su tensión eléctrica, su presión barométrica, la disminución de la gravitación, la intensidad del color azulado celeste, la debilidad de la luz durante su paso por las capas sobrepuestas al aire, las refracciones horizontales y el calor del agua hirviendo en diferentes alturas". (Tomo I, pg. xxxij).

En estas afirmaciones, es importante hacer precisiones conceptuales sobre el fenómeno eléctrico, cuando se afirma en uno de sus libros: "La superficie radiante del globo, y la carga eléctrica de las bajas regiones de la atmósfera, que varía según la naturaleza del suelo y el yacimiento de los continentes y los mares..." (Traducción del libro de Humboldt por L. Alvarado, Tomo II, pg 239). En la época de Humboldt se hablaba de "fluido eléctrico" o "fuego eléctrico", no de "carga eléctrica", pues este concepto aparece a finales del siglo 19 cuando Thompson descubre el electrón en 1897. Este puede ser un error de traducción.

En otro de sus escritos Humboldt hizo excelentes observaciones de correlación entre los rayos y el movimiento solar cuando escribe: "Según la latitud de Cumanacoa, el sol pasa por su zenit la primera vez el 16 de abril y la segunda el 27 de agosto. Por lo que acabamos de exponer se advierte que estos dos pasos coinciden con el principio de las lluvias y de las grandes explosiones eléctricas...” (Tomo II, pg. 53).

En nuestra investigación sobre rayos en la zona tropical hemos hecho campañas de medición multianual y hemos encontrado correlación con el fenómeno meteorológico de desplazamiento de la Zona de Confluencia Intertropical (ZCIT) (2002). Por ejemplo, en la figura 3 se presenta la variación multianual en la ciudad de Bogotá, Colombia, localizada en la región andina y la ciudad de la Habana, Cuba, localizada en la región Caribe. Mientras Bogotá presenta un comportamiento bimodal, en la Habana es monomodal, de acuerdo con el desplazamiento de la ZCIT. Interesante lograr una explicación del comportamiento de la actividad de rayos en función con el movimiento solar.

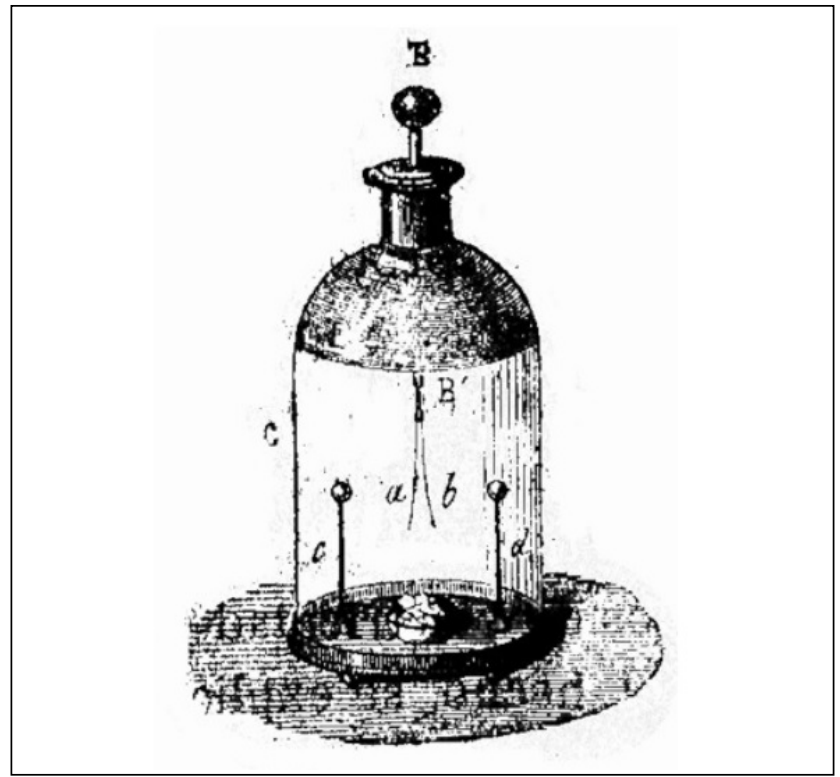

Figura 2. Electroscopio para determinar la naturaleza de la electricidad. Tomado de: http://www.librosmaravillosos.com/ laelectricidadysusmaravillas/index.html )

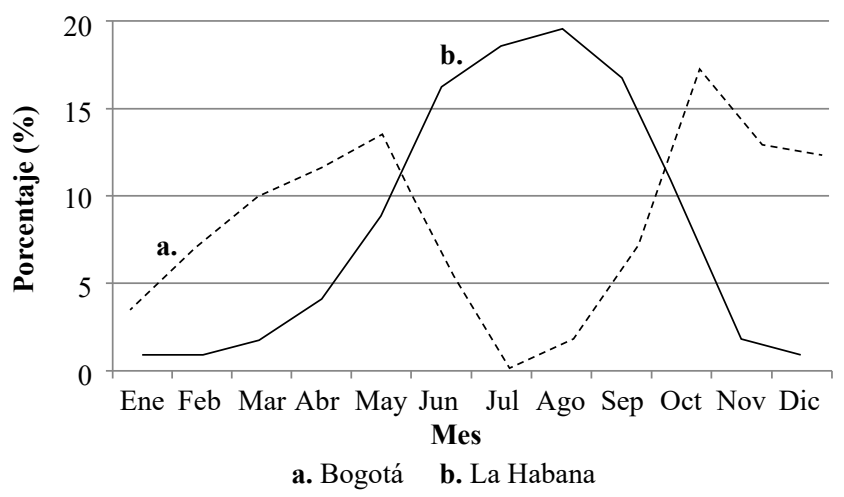

Figura 3. Comportamiento bimodal y monomodal de la actividad de rayos en el trópico. (Tomado de Torres, 2002) 
Así mismo, Humboldt hizo excelentes observaciones de correlación entre temblores-viento-rayos-temperatura-sol y medición de polaridad, cuando escribe: "Algunos minutos antes del primer sacudimiento ocurrió un viento violentísimo seguido de una lluvia eléctrica de goterones. Tanteé al punto la electricidad atmosférica con el electrómetro de Volta. Las bolillas se apartaban 4 líneas; la electricidad pasó con frecuencia del positivo al negativo, como sucede durante las tormentas, y en el Norte de Europa, y aun a veces con la caída de la nieve". (Traducción del libro de Humboldt por L. Alvarado, pg. 221, Vol. II).

Sin embargo, no tuvo el interés de profundizar en estas observaciones y plantear hipótesis sobre tales fenómenos, cuando escribe: "Lejos estoy de buscar la causa fisica de estas diferencias en las únicas modificaciones de la carga eléctrica del aire: debo, no obstante, añadir que algo al Este de La Guaira, de la parte de Macuto, distante de las casas y a más de cien toesas de distancia de las rocas de gneis, he podido apenas obtener, durante varios dias, algunas débiles señales de electricidad positiva, cuando a las mismas horas de la tarde, con el mismo electrómetro de Volta armado de una mecha fumante, habia observado en Cumaná una separación de 1 - 2 líneas de las bolillas de saúco. Expondré más abajo las variaciones regulares que a diario experimenta la tensión eléctrica del aire en la zona tórrida, las cuales indican una sorprendente correlación entre las variaciones de la temperatura y la altura del sol". (Traducción del libro de Humboldt por L. Alvarado, pgs. 267-268, Vol. I).

Así mismo hizo observaciones entre el fenómeno eléctrico y los volcanes y temblores: "El 4 de noviembre tuvimos un violento temblor de tierra, que felizmente no causó mayor perjuicio. Me admiró ver que la inclinación magnética disminuyó en 1,1 $1^{\circ}$ durante ese acontecimiento". (Traducción del libro de Humboldt por L. Alvarado, pg. 417, Vol. II). Es interesante observar que hoy en día se está trabajando en la predicción de los movimientos de tierra (geología) con la medición de fenómenos electromagnéticos.

El rayo del Catatumbo, piratas e independencia. Anecdóticamente el rayo del Catatumbo frustró en 1595 un ataque del pirata inglés Francis Drake a la ciudad de Maracaibo y fue narrado en un bello episodio en la obra épica "La Dragontea", de Lope de Vega, en la que relata cómo la luminosidad desplegada por el relámpago le permitió a un vigía divisar las naves de Drake y alertar a la guarnición, que logró repeler el ataque nocturno.

Además, durante la guerra de independencia, el rayo del Catatumbo sirvió de faro para la fuerza naval del Almirante neogranadino José Prudencio Padilla, quien logró derrotar a los navíos españoles el 24 de julio de 1823 en la batalla naval del lago de Maracaibo.

Humboldt y el rayo del Catatumbo. “¿Qué cosa es el fenómeno luminoso conocido con el nombre de farol de Maracaybo que todas las noches se ve del lado del mar como en lo interior del país, por ejemplo en Mérida, donde el señor de Palacio lo ha observado durante dos años? La distancia de más de 40 leguas a que se distingue la luz ha hecho creer que podría ser el efecto de una tempestad o de explosiones eléctricas que tuviesen lugar diariamente en una garganta de montañas y aun se asegura que se oye el ruido del trueno cuando se aproxima uno al farol. Otros pretenden vagamente que esto es un volcán de aire y que terrenos asfálticos parecidos a los de Mena, causan exhalaciones inflamables y tan constantes en su aparición. El sitio en que este fenómeno se presenta es un país montañoso e inhabitado en las orillas del río Catatumbo, cerca de su unión con el río Sulia. La posición del farol es tal que, situado casi en el meridiano de la boca de la laguna de Maracaybo, dirige a los navegantes como un fanal". [Humboldt, Alejandro, y Bonpland, Aimé, Viage, Vol. 2, Lib. V, Cap. XVI, p. 390, nota.]

Con un análisis de su recorrido y sus escritos, es fácil conocer la relación de Humboldt con el rayo del Catatumbo, pues no se trasladó al occidente de Venezuela ni observó directamente el "rayo del Catatumbo" ni hizo medición alguna, según sus escritos.

Lo más próximo que estuvo de este fenómeno atmosférico fue primero en Caracas (a $700 \mathrm{~km}$ ), en Cumaná (a $820 \mathrm{~km}$ ) y luego, en su recorrido por el río Magdalena, estuvo a más de $200 \mathrm{~km}$ del lago de Maracaibo en línea recta. Esto parece indicar que tomó información de Manuel Palacio-Fajardo (así lo describe en el Vol. 2 de su Viage), de Boussingault y de Agustín Codazzi.

Manuel Palacio Fajardo, prócer de la Independencia, al que se refiere Humboldt en sus escritos, escribió en 1817: "Entramos a San Antonio de Cúcuta en donde el Congreso de la República había promulgado la Constitución. Nos quedamos alli [Cúcuta] hasta el 5 de mayo y durante las tres noches que pasamos en esta ciudad volvimos a ver, hacia el norte, la luz del "farol" del río Catatumbo. Esta luz lejana no tiene la apariencia de rayos ni en ella se distingue los centelleos y el zig-zag del relámpago; es como un vapor luminoso, muy fugaz que abarca un gran espacio y aparece y desaparece con suficiente rapidez para hacer creer que es permanente"

Jean-Baptiste Joseph Dieudonné Boussingault fue un notable científico francés creador de la química orgánica y la agronomía experimental que realizó numerosas observaciones barométricas y botánicas. En Santa Fé de Bogotá se incorporó al ejército de Bolívar y viajó ampliamente por el norte del subcontinente sudamericano después de 1822 .

En las Cartas Americanas de Humboldt publicadas por la Fundación Biblioteca Ayacucho (1989, 308 páginas), se tienen las notas sobre Humboldt escritas por Boussingault:

"...Humboldt se interesaba vivamente en nuestra expedición*. No solo íbamos a recorrer los lugares que él habia visitado hacia veinte años, sino que pensábamos quedarnos (do modo que) muchas observaciones hechas por el debian ser completadas y ampliadas".

Boussingault había sido alistado por el botanista Antonio Zea, enviado a Europa por Bolívar en misión política. Entre otras cosas, Zea debía reunir jóvenes instruidos 
y emprendedores para fundar en Santa Fe de Bogotá, capital de la nueva república, establecimientos científicos particularmente destinados a formar ingenieros civiles y militares. Boussingault partió con Roulin, Rivero y algunos más."

Aporte de Boussingault a la ciencia: fijación del $N_{2}$ El nitrógeno $(\mathrm{N})$, se encuentra formando parte de la atmósfera, integrando un $78 \%$ de la misma. Es un elemento bastante inerte, no reacciona fácilmente con otros compuestos, así que antes de ser aprovechado por la mayoría de los seres vivos, debe pasar por un proceso de fijación, el cual está definido como su combinación con oxígeno o hidrógeno.

La fijación natural (abiótica) generalmente ocurre por procesos químicos espontáneos, como la oxidación que se produce por la acción de los rayos, que forma óxidos de nitrógeno a partir del nitrógeno atmosférico y posteriormente nitratos.

Hoy en día se conoce científicamente que los rayos, cuando fijan el nitrógeno al impactar en el suelo son un verdadero banquete para las plantas, que se refleja rápidamente en un vigor y crecimiento extraordinarios.

Agustín Codazzi: informante del rayo del Catatumbo. Agustín Codazzi, en su libro Resumen de la Geografía de Venezuela (1841), relata sobre el rayo del Catatumbo:

"En los lugares en que desemboca el Catatumbo, parece que se acumulan más frecuentemente los chubascos, y allí el trueno y los relámpagos asustan continuamente al navegante: parece que la materia eléctrica está concentrada en aquellos parajes, en los cuales se observa todas las noches un fenómeno luminoso que es como un relámpago que de tiempo en tiempo enciende el aire. Desde la mar se mira como si estuviese sobre la isla de Toas, que está casi en el meridiano de la barra de Maracaibo: pasa sobre las bocas del Catatumbo y sirve de guía a los marinos".

"Un relámpago continuado que parece surgir en la región pantanosa del río Zulia, Catatumbo y sus alrededores".

Certezas sobre el rayo hoy en día. En toda actividad de ciencia y tecnología es demostrable que no hay verdades absolutas sino certezas temporales. Un ejemplo de ello han sido los parámetros del rayo medidos por Berger entre la década de 1950 y 1970, que han servido como base para el desarrollo de las grandes investigaciones de rayos en el mundo, y en normalización internacional, que hoy son cuestionados con rigurosidad por mediciones directas de parámetros del rayo en la misma zona templada donde fueron medidos hace más de 50 años, así como por mediciones en zona tropical.

Las cinco certezas que influencian la actividad de rayos en una zona como el Catatumbo (Torres, 2015) son:

- Vientos. La actividad de rayos permanentes es ocasionada por la circulación de sistemas de vientos, cuyas nubes de tormenta transportan cargas eléctricas en su interior.

- Orografía. El sistema montañoso de la región que interactúa con el sistema de vientos, produciendo concentración de nubes de tormenta y facilidad para generar rayos por el fenómeno de convección.

- Suelo. Material sobre la superficie del terreno. La superficie está ligada a otro factor que tiene que ver con los cambios por actividad humana.

- Subsuelo. La posibilidad de la existencia de algún agente causal en el subsuelo, por ejemplo, fuentes geomagnéticas o radiactivas que pueden producir cargas iónicas en el entorno.

- Cambios por actividad humana. Se refiere al termino de efecto isla por calentamiento urbano.

De acuerdo con las investigaciones realizadas en Colombia (Torres, 2015) y discutidas ante la comunidad académica internacional y mediciones satelitales de la NASA, el Catatumbo es la región de mayor actividad de rayos del mundo, con los siguientes sitios localizados en la región colombiana (Tabla 1):

Graficando estos puntos que hoy en día tenemos certezas sobre su actividad para la región del Catatumbo, se tiene el mapa de la figura 4.

Tabla 1. Máxima densidad de rayos en Suramérica. (Adaptado de Torres, 2016)

\begin{tabular}{|c|c|c|c|c|c|}
\hline Rango Global & $\begin{array}{c}\text { Densidad } \\
\text { Rayos } / \mathbf{k m}^{2}\end{array}$ & Lat $\left({ }^{0}\right)$ & Long $\left(^{0}\right)$ & $\begin{array}{l}\text { Sitio más cercano en } \\
\text { Suramérica }\end{array}$ & País \\
\hline 1 & 232 & 9,75 & $-71,65$ & Lago Maracaibo & Venezuela \\
\hline 4 & 172 & 7,55 & $-75,35$ & Cáceres & Colombia \\
\hline 7 & 139 & 8,85 & $-73,05$ & El Tarra & Colombia \\
\hline 11 & 124 & 5,75 & $-74,95$ & Norcasia & Colombia \\
\hline 18 & 114 & 8,45 & $-74,55$ & Majagual & Colombia \\
\hline 25 & 106 & 8,15 & $-76,85$ & Turbo & Colombia \\
\hline 46 & 95 & 11,15 & $-72,95$ & Barrancas & Colombia \\
\hline 74 & 88 & $-17,25$ & $-65,05$ & Chimoré & Bolivia \\
\hline 78 & 88 & 10,35 & $-70,95$ & El Corozo & Venezuela \\
\hline 136 & 77 & 10,45 & $-70,35$ & Santa Rosa & Colombia \\
\hline
\end{tabular}




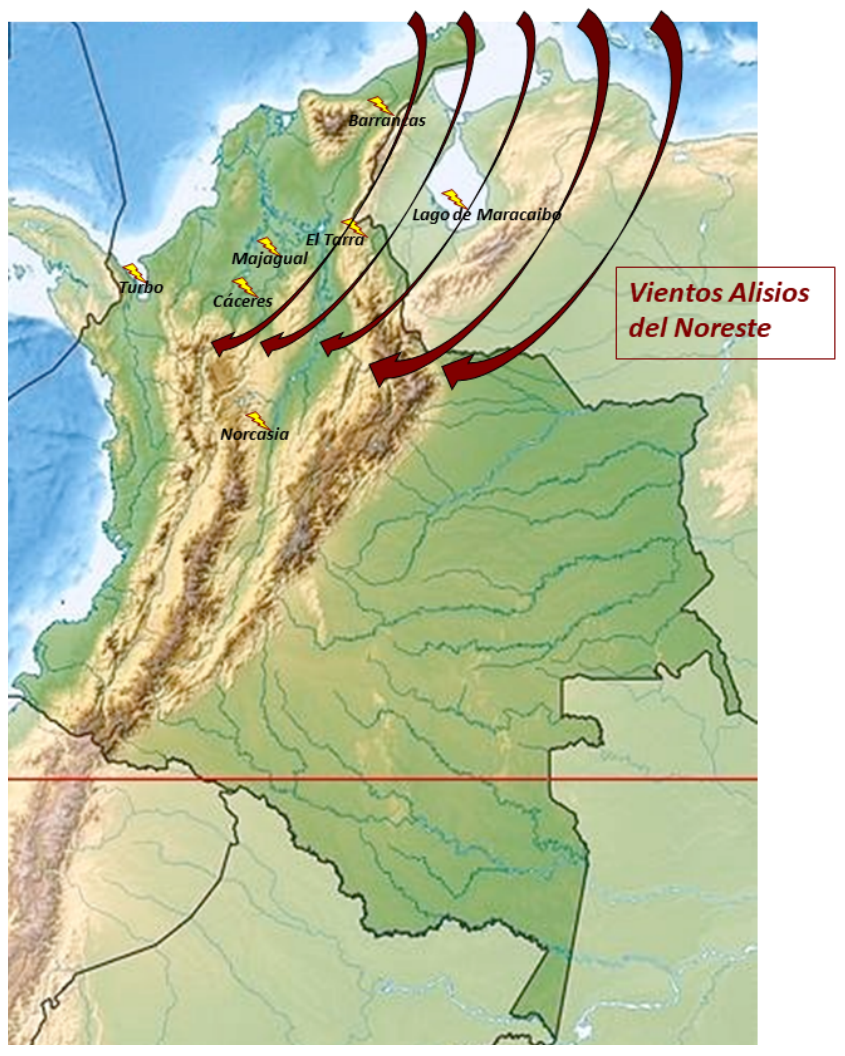

Figura 4. Actividad de rayos en la zona de Catatumbo (Elaboración propia)

\section{Conclusiones}

Si bien Humboldt no visitó directamente la zona del Catatumbo, sus informantes lograron una excelente descripción del rayo del Catatumbo. Solo faltó que Humboldt planteara una explicación física, científica, que ya estaba latente en sus observaciones escritas:
- “Otros pretenden vagamente que esto es un volcán de aire”, es decir los vientos alisios del Noreste.

- "[...] podría ser el efecto de una tempestad o de explosiones eléctricas que tuviesen lugar diariamente en una garganta de montañas". El estrechamiento de las montañas (orografia) es una explicacion que junto con otros factores (vientos, suelo y subsuelo) dan explicacion sobre la actividad de rayos en la region del Catatumbo.

- "[...] que varía según la naturaleza del suelo y el yacimiento de los continentes y los mares". Los otros factores involucrados: el suelo y el subsuelo.

\section{Conflicto de intereses}

Ninguno que declarar

\section{Referencias}

Humboldt A. Viage a las regiones equinocciales del nuevo continente, 1799-1804, Vols. I, II, III, IV, V. 797 paginas. Escrito en París, del año 1804 a 1827.

Humboldt et Bonpland. Des voyages dans les régions équatoriales du nouveau continent fait dans les années 1799 a 1804, traducidos por el venezolano Lisandro Alvarado de 1941 a 1956.

Agustín Codazzi. (1841) Resumen de la Geografía de Venezuela, Fournier, Paris, pp. 20, 464 y 466.

Torres, H. (2002). El Rayo, Mito, Ciencia y Tecnología, ISBN: 958-70-1213-5, Unibiblos, Bogotá.

Torres, H. (2015). El rayo en el trópico, certezas temporales de investigación sobre el fenómeno del rayo. ISBN 978-958775-454-4 Colección apuntes maestros, Ed. UN, Bogotá, Colombia.

Albrecht, R, et. al. (2016) "Where are the lightning hotspots on Earth?", Bull. Amer. Meteor. Soc. doi: 10.1175/ BAMS-D-14-00193.1.

Torres, H. (2018). El enigma del electromagnetismo bajo la lupa ISBN 978-620-2-15081-1. Editorial Académica Española EAE Publishing LAP LAMBERT Academic Publishing GmbH\& Co. KG. 